\title{
Large-scale structure of brown rat (Rattus norvegicus) populations in England: Effects on rodenticide resistance
}

Mohd Z.H Haniza, Sally Adams, Eleanor P Jones, Alan MacNicoll, Eamonn B Mallon, Robert H Smith, Mark S Lambert

The brown rat, Rattus norvegicus is a relatively recent ( $<300$ years) addition to the British fauna, but by association with negative impacts on public health, animal health and agriculture, it is regarded as one of the most important vertebrate pest species. Anticoagulant rodenticides were introduced for brown rat control in the 1950s and are widely used for rat control in the UK, but long-standing resistance has been linked to control failures in some regions. One thus far ignored aspect of resistance biology is the population structure of the brown rat. This paper investigates the role population structure has on the development of anticoagulant resistance. Using mitochondrial and microsatellite DNA, we examined 186 individuals (from 15 counties in England and one location in Wales near the Wales-England border) to investigate the population structure of rural brown rat populations. We also examined individual rats for variations of the VKORCI gene previously associated with resistance to anticoagulant rodenticides. We show that the populations were structured to some degree, but that this was only apparent in the microsatellite data and not the mtDNA data. We discuss various reasons why this is the case. We show that the population as a whole appears not to be at equilibrium. The relative lack of diversity in the mtDNA sequences examined can be explained by founder effects and a subsequent spatial expansion of a species introduced to the UK relatively recently. We found there was a geographical distribution of resistance mutations, and relatively low rate of gene flow between populations, which has implications for the development and management of anticoagulant resistance. 
1 Large-scale structure of brown rat (Rattus norvegicus) populations in England: Effects on 2 rodenticide resistance.

The brown rat, Rattus norvegicus is a relatively recent ( $<300$ years) addition to the British fauna, but by association with negative impacts on public health, animal health and agriculture, it is regarded as one of the most important vertebrate pest species. Anticoagulant rodenticides were introduced for brown rat control in the 1950s and are widely used for rat control in the UK, but long-standing resistance has been linked to control failures in some regions. One thus far ignored aspect of resistance biology is the population structure of the brown rat. This paper investigates the role population structure has on the development of anticoagulant resistance. Using mitochondrial and microsatellite DNA, we examined 186 individuals (from 15 counties in England and one location in Wales near the Wales-England border) to investigate the population structure of rural brown rat populations. We also examined individual rats for variations in the VKORC1 gene previously associated with resistance to anticoagulant rodenticides. We show that the populations were structured to some degree, but that this was only apparent in the microsatellite data and not the mtDNA data. We discuss various reasons why this is the case. We show that the population as a whole appears not to be at equilibrium. The relative lack of diversity in the mtDNA sequences examined can be explained by founder effects and a subsequent spatial expansion of a species introduced to the UK relatively recently. We found there was a geographical distribution of resistance mutations, and relatively low rate of gene flow between populations, which has implications for the development and management of anticoagulant resistance. 
21 Mohd Z.H. Haniza 1, Sally Adams ${ }^{2}$, Eleanor P. Jones ${ }^{3}$, Eamonn B. Mallon ${ }^{4}$, Alan D. MacNicoll

22 5, Robert H. Smith ${ }^{6} \&$ Mark S. Lambert ${ }^{5 *}$

$23{ }^{1}$ Faculty of Science and Mathematics, Universiti Pendidikan Sultan Idris, Malaysia

$24{ }^{2}$ School of Life Sciences, University of Warwick, U.K.

$25{ }^{3}$ Fera Science Ltd., Sand Hutton, York, U.K.

$26{ }^{4}$ Department of Biology, University of Leicester, U.K.

$27{ }^{5}$ Animal and Plant Health Agency, Sand Hutton, York, U.K.

$28{ }^{6}$ School of Applied Sciences, University of Huddersfield, U.K.

29

30

$31 *$ Corresponding author

32 Mark Lambert

33 Animal and Plant Health Agency

34 Sand Hutton

35 York YO41 1LZ

36 mark.lambert@apha.gsi.gov.uk

37 Tel: 01904406604

38 Fax: 01904462111 


\section{INTRODUCTION}

42 The brown rat (Rattus norvegicus) first arrived in Britain in the early $18^{\text {th }}$ century, originally

43 from the steppes of Central Asia (Yalden 1990), and is regarded as one of the most important

44 vertebrate pest species in the UK (Buckle \& Smith 2015). Brown rats are associated with risks to

45 public health, animal health, and impacts on agriculture, infrastructure and native wildlife

46 (Meehan 1984; Webster et al. 1995). Anticoagulant rodenticides have been widely used for

47 controlling rat populations in the UK since the introduction of warfarin in the 1950s (Hayes

48 1950). However, resistance to warfarin was encountered by 1960 (Boyle 1960), and it is thought

49 that chance genetic mutations arise that confer heritable resistance to anticoagulant compounds;

50 intensive use of anticoagulants applies a selection pressure that increases the frequency of

51 resistant rats within a population (Greaves 1995; Greaves \& Ayres 1967; Smith \& Greaves

52 1987). In response to the emergence of warfarin resistance, 'second-generation' anticoagulant

53 rodenticides, including bromadiolone and difenacoum, were developed that controlled warfarin-

54 resistant rats (Hadler \& Shadbolt 1975; Hadler et al. 1975), although some rat populations have

55 now evolved resistance to some of these more toxic anticoagulants (Lund 1985; Redfern \& Gill

56 1978).

58 Warfarin acts by interfering with blood coagulation (blood clotting). Several factors in the

59 coagulation process are dependent on sufficient vitamin $\mathrm{K}$ levels for their proper functioning

60 (Suttie 1980). Vitamin K hydroquinone is an essential cofactor for post-translational gamma-

61 carboxylation of these blood coagulation factors (Sadowski et al. 1976). During each

62 carboxylation step, one molecule of vitamin $\mathrm{K}$ hydroquinone is oxidized to vitamin $\mathrm{K} 2,3$

63 epoxide. The recycling of this micronutrient is carried out by the vitamin $\mathrm{K}$ epoxide reductase 
64 (VKOR) complex. Suppression of the VKOR by anticoagulants inhibits the carboxylation of

65 clotting factors and thus compromises the coagulation process (Bell \& Caldwell 1973). Early

66 studies showed that warfarin resistance was inherited in rats as a single autosomal dominant gene

67 (Rw) (Greaves \& Ayres 1967). More recently it has been demonstrated that mutations or

68 variations (Single Nucleotide Polymorphisms) in a gene of the VKOR complex, VKORC1, are

69 involved in the resistance to anticoagulants in rats (Pelz et al. 2005; Rost et al. 2004). These

70 variations, which result in amino acid substitutions in the protein VKORC1, may decrease the

71 sensitivity of the protein to warfarin, maintaining the efficiency of the coagulation process in

72 warfarin-exposed resistant rats (Pelz et al. 2005).

74 It has been suggested that rodenticide resistance spread in European rat populations from initial 75 focal points, with resistant rats expanding into new areas (Pelz et al. 2005). Earlier work noted 76 that resistance spread at about 5km per year (Brodie 1976; Drummond 1970). However, warfarin 77 resistance, though widespread, is not ubiquitous. One possible explanation for this pattern is that 78 gene flow between different rat populations is limited. The rate at which resistance is acquired is 79 a function of the resistance allele's frequency, its dominance, the relative fitness of being 80 resistant and, often overlooked, the pest organism's population structure (Roush \& McKenzie 81 1987). Population structure, the subdivision of populations into smaller interbreeding units, is 82 important as it controls gene flow from area to area. Gene flow has two effects on the spread of 83 resistance. Firstly, the greater the gene flow between areas, the more likely resistance genes are 84 to spread. Secondly, if resistance genes spread into areas where pesticides are not used, 85 resistance genes may be diluted by susceptible individuals (Wool \& Noiman 1983). In this study, 86 we used microsatellites, which are informative of population level structures and gene-flow 
87 between populations (e.g. Balloux and Lugon-Moulin, 2002; Hutchison and Templeton, 1999), 88 and mitochondrial DNA sequences, which can provide information about older events in the 89 colonisation history of the rats in the UK, as has been done for, for example, house mice (Searle 90 et al. 2009).

91

92 We examined 186 rural brown rat DNA samples (184 from England and two from a location in 93 Wales near to the Wales-England border) in order to investigate population structure through 94 mitochondrial DNA (mtDNA) and microsatellite analysis. We also examined the distribution of 95 VKORC1 variants within the sampled populations. The samples were collected opportunistically 96 and hence sampling was not geographically stratified, nor was regional coverage comprehensive,

97 however the results reveal novel insights into the structure of rural brown rat populations that 98 have important implications for the development and management of anticoagulant resistance. 
100 MATERIALS AND METHODS

\section{Sample details}

102 We analysed 184 rats from 15 counties in England; we also included two rats collected from

103 Welshpool, Powys, 6km from the Wales-England border (Figure 1). The majority of the samples

104 came from an extensive trapping program carried out by the Animal and Plant Health Agency's

105 (APHA) National Wildlife Management Centre (then part of the Central Science Laboratory),

106 York from 1990-2000. These samples were obtained as livers stored at $-20^{\circ} \mathrm{C}$. Rats were also

107 trapped on several farms in Leicestershire and Yorkshire during 2004 and 2005 and stored at -

$10820^{\circ} \mathrm{C}$. In addition, samples from Cambridge were obtained from rats trapped in 2004 as extracted

109 DNA from the Babraham Institute, Cambridge. All research that involved the use of live animals

110 was approved in advance by written confirmation from the APHA's Animal Welfare and Ethical

111 Review Body (AWERB) or equivalent at the time of the study. Our AWERB membership has

112 both internal and external members including lay representatives and experts in a variety of

113 apposite areas such as veterinary surgery, statistics and animal welfare.

\section{DNA extraction}

116 Total genomic DNA was extracted from either $20 \mu \mathrm{g}$ of liver or a $15 \mathrm{~mm}$ tail tip using the

117 Wizard SV Genomic DNA Purification System (Promega) as per instructions. DNA samples 118 were stored at $-20^{\circ} \mathrm{C}$ until use.

\section{Mitochondrial DNA (mtDNA)}

121 For each individual, a 425 base pair region of the hypervariable region 1 (HVR1) segment within 122 the mtDNA control region was PCR amplified. PCR was carried out as per Hingston et al. (2005) 
123 with slight modifications. Primers used were L283 (5'-TACACTGGTCTTGTAAACC-3') and

124 H16498 (5'-CCTGAAGTAGGAACCAGATG-3'). A $20 \mu$ reaction was used in which $2 \mu 1$

125 genomic DNA was added to the reaction mixtures containing $10 \mu 1$ PCR reaction mix (YorkBio),

$1261 \mu \mathrm{l}(10 \mathrm{uM})$ of each primer and $6 \mu \mathrm{l}$ of $\mathrm{H}_{2} \mathrm{O}$. PCR was carried out on a T1 Thermocycler

127 (Biometra, Goettingen, Germany). The PCR conditions were: 30 cycles of denaturation at $94^{\circ} \mathrm{C}$

128 for $45 \mathrm{~s}$, annealing at $50^{\circ} \mathrm{C}$ for $45 \mathrm{~s}$, elongation at $72^{\circ} \mathrm{C}$ for one min, and a final extension at $72^{\circ} \mathrm{C}$

129 for $30 \mathrm{~min}$.

130

131 PCR products were then cleaned with the YorkBio PCR Cleanup kit to remove any

132 unincorporated nucleotides and primers that can interfere with the sequencing process. The

133 cleaned PCR products were sequenced by the John Innes Genome laboratory using an ABI3700

134 capillary sequencer.

\section{Microsatellite DNA}

137 We used six microsatellite loci from the literature, that are highly variable for brown rats (D3,

138 D5, D8, D12, D16, D17) (Heiberg 2002). The PCR volume was 10ul, containing 5ul of PCR

139 reaction mix (YorkBio), $0.5 \mathrm{ul}$ of DNA, $0.5 \mathrm{ul}$ of each primer and 3.5ul of $\mathrm{ddH}_{2} \mathrm{O}$. One primer of

140 each locus was labelled with one of the fluorescent dyes, PET, 6FAM or VIC. PCR was run on a

141 T1 Thermocycler (Biometra, Goettingen, Germany) using the following conditions: one cycle of

142 denaturation at $94^{\circ} \mathrm{C}$ for five mins, 35 cycles of denaturation $\left(94^{\circ} \mathrm{C}\right.$ for $\left.30 \mathrm{~s}\right)$, annealing $\left(50^{\circ} \mathrm{C}\right.$ for

$14330 \mathrm{~s})$ and elongation $\left(72^{\circ} \mathrm{C}\right.$ for $\left.30 \mathrm{~s}\right)$. Finally, there was one cycle of extension at $72^{\circ} \mathrm{C}$ for $30 \mathrm{~s}$.

144 PCR products were run as two batches on an ABI 3730 capillary sequencer (Applied 
145 Biosystems, Massachusetts, USA). The results were scored using GeneMapper v0.5.0 (Applied

146 Biosystems).

147

\section{VKOR analysis}

149 Pelz et al. (2005) identified seven different VKORC1 variants in warfarin resistant Norway rats;

150 four of these (Tyr139Cys, Leu120Gln, Leu128Gln and Tyr139Ser) were reported from the

151 United Kingdom (Table 1), we therefore analysed our samples for the presence of these four

152 variants. A sub-sample of the rats had previously been screened for resistance to warfarin using a

153 blood clotting response (BCR) test; individuals which were determined by the BCR test to be

154 warfarin resistant ('BCR positives') but were found to have none of the above VKORC1 variants

155 were screened for other VKOR polymorphisms by genetic sequencing. PCR primers and detailed 156 protocols were as per Pelz et al. (2005). All PCR products were visualized on a 3\% agarose gel. .

158 ARMS-PCR

159 Amplification refractory mutation system (ARMS)-PCR was used to detect the Tyr139Cys 160 variant. This technique employs two primer pairs to amplify, respectively, the two different 161 alleles of a single nucleotide polymorphism (SNP) in a single PCR reaction.

162

163 Restriction fragment length polymorphisms

164 The other three VKORC1 variants create novel restriction sites in exon 3, which allow one of the

165 three enzymes (Stu I, Bsr I, Mnl I) to cut the fragment at this point creating fragments of 166 characteristic sizes (Table 1). 


\section{Data Analysis}

170 For the mtDNA analyses, East Sussex and West Sussex were combined (such that for Sussex, $\mathrm{n}$

$171=12$ ), Shropshire and Powys were combined (such that for Shropshire + Powys $n=6$ ). We

172 analysed the phylogenetic relationship among the mtDNA haplotypes using two methods. First, a

173 minimum spanning network based on a matrix of the observed nucleotide differences was

174 calculated using the program ARLEQUIN 3.01 (Excoffier et al. 2005). Second, the genetic

175 distance between haplotypes was calculated assuming a Tamura \& Nei model of sequence

176 evolution (Tamura \& Nei 1993). These distances were then used to construct a neighbour-joining

177 tree using the computer program SPLITSTREE (Huson 1998); 1,000 bootstrap replicates were

178 calculated to estimate the support for each node in both the minimum spanning network and the

179 neighbour-joining tree. For comparison, we included Genbank sequences from several laboratory

180 rat strains (Wistar, BN/SsNHsdMCW, F344 X BN F1 and Sprague-Dawley), a wild caught

181 brown rat from Denmark, and as an outgroup, a Rattus rattus sequence.

182

183 The geographic distribution of genetic variation was estimated using Analysis of Molecular

184 Variance (AMOVA) performed by ARLEQUIN 3.01. Gene diversity $(h)$ and nucleotide diversity

$185(\pi)$ of the various populations and their respective standard deviations were calculated using

186 ARLEQUIN 3.01. ARLEQUIN 3.01 was also used to perform mismatch analysis to compare the

187 distribution of the observed number of differences between pairs of haplotypes (mtDNA) and the 188 expected distribution under various models of population change (Rogers \& Harpending 1992). 
190 Geographical distances were calculated as the distance from the central national grid reference of

191 one population area to the central national grid reference of the other. This distance was

192 compared with $\Phi_{\text {st, }}$, an analogue of Wright's $F_{\text {st }}$ statistic (Wright 1951), using a Mantel test

193 carried out with the computer program GenAlEx 6 (Peakall \& Smouse 2006). Observed and

194 expected heterozygosities were calculated for the microsatellite data in ARLEQUIN 3.01.

195

196 For the microsatellite analyses, East Sussex and West Sussex were combined (such that for

197 Sussex, $n=12)$, Powys and Shropshire were combined $(n=6)$; samples from Greater London ( $\mathrm{n}$

$198=3)$ and Gloucestershire $(n=3)$ were not included (because of the small sample sizes). Using

199 microsatellite data, inferences on the number of populations were made with the fully Bayesian

200 clustering method implemented in STRUCTURE 2.1 (Pritchard et al. 2000). With the aim of

201 determining the most likely number $K$ of population units, the program was run ten times for

$202 K=2$ to $K=10$. The model with admixture has been used with correlated frequencies. After

203 some preliminary tests of the convergence time needed for the Monte-Carlo Markov chain, a

204 burn-in period of 100,000 steps followed by 1,000,000 steps was used. The most likely value of

$205 \mathrm{~K}$ was considered using the maximum log likelihood values for the aggregated runs, and by

206 considering Delta $\mathrm{K}$, the rate of change in the log-likelihood values between the values of $\mathrm{K}$

207 (Evanno et al. 2005), calculated in Structure Harvester (Earl \& vonHoldt 2012). Structure plots

208 were created for $K=3-5$ using the programs CLUMPP (Jakobsson \& Rosenberg 2007) and

209 DISTRUCT (Rosenberg 2004), which aggregate and plot out the multiple runs for each value of

$210 K$ into single outputs. 


\section{RESULTS}

\section{Molecular diversity}

214 A total of six unique mtDNA haplotypes were represented in the individuals sampled. The

215 nucleotide differences between haplotypes are shown in Table 2. Sequences representing each

216 unique mtDNA haplotype have been deposited in Genbank under accession numbers DQ897633-

217 DQ897638. There are 10 variable nucleotide positions all of which are transitions (Table 2),

218 forming two haplogroups. These are RNH1, the most common haplotype, and RNH6, which

219 diverges from it by a single mutation, RNH2, the second most common haplotype, and RNH5 220 and RNH3, which diverge from it by single mutations with a further haplotype RNH3, two

221 mutations from RNH5 (Figure $2 \mathrm{a}$ and $\mathrm{b}$ ). Figure $2 \mathrm{~b}$ also includes several other sequences

222 obtained from Genbank for this region of the R. norvegicus mitochondrial genome. These 223 include sequences from several laboratory rat strains (Wistar, BN/SsNHsdMCW, F344 X BN F1

224 and Sprague-Dawley), a wild caught brown rat from Denmark, and as an outgroup, a Rattus 225 rattus sequence. Relative to our samples, the Wistar strain has a deletion at position 77 , the $R$. 226 rattus and Sprague-Dawley strain have an insertion at position 305. The $R$. rattus sample also 227 has an insertion at position 266. Neither haplogroup (nor haplotype) dominated any region, nor 228 was there any geographic structuring to the haplotype distributions. The haplotypic $(h)$ and 229 nucleotide diversity $(\pi)$ indices are given in Table 3 . These values range from 0 (monomorphic 230 populations in Leicestershire, Dorset, Hampshire and Greater London) to $h=0.67$ and $\pi=$ 2310.0118 in Gloucestershire, with an overall average of $0.44+/-0.03$ for $h$ and $0.0069+/-0.0040$ 232 for $\pi$. Figure 3 shows the observed number of differences between pairs of haplotypes 233 (mismatch analysis). This distribution is not significantly different from the spatial expansion 234 model; $\mathrm{SSD}=0.1023$, Bootstrap replicates $=1,000, \mathrm{p}=0.133$ (Rogers \& Harpending 1992). 
236 Eighty-one alleles were found across six microsatellite loci tested in all individuals. All of the

237 loci were polymorphic and the number of alleles ranges from 10 (D5) to 18 (D12). The mean

238 number of alleles detected at each locus was 13.5. The observed heterozygosities (Ho) and

239 expected heterozygosities $(\mathrm{He})$ are shown in Table 4.The highest observed heterozygosity was

240 seen in locus D8 in the Dorset population and locus D3 in the Worcester population, and the

241 lowest was locus D5 in the Worcester population. Only $22.6 \%$ of the Ho were higher than He,

$2421.2 \%$ of Ho were the same as He (locus D5 from the Wiltshire population) and 76.2\% of Ho

243 were lower than He. A substantial deficit of heterozygotes was observed for locus D17 in the

244 Berkshire, Leicestershire, Wiltshire and Yorkshire populations, locus D5 in the Sussex and

245 Worcestershire populations, and locus D8 in the Worcestershire population.

247 Genetic structure

248 Mitochondrial DNA

249 A result was not obtained for one sample from Kent; hence 185 samples were included in the 250 analyses. AMOVA $(\mathrm{p}<0.001)$ showed that $33.4 \%$ of the total variance was assigned to between 251 population (county) diversity $(\mathrm{df}=13$, sum of squares $=105.9$, variance components $=0.55)$ 252 while $66.6 \%$ was attributed to diversity within populations $(\mathrm{df}=170$, sum of squares $=186.02$, 253 variance components $=1.09)$

Microsatellite DNA

256 A result was not obtained for one sample from Sussex; hence 179 samples were included in the 257 analyses (Greater London and Gloucestershire were not included). AMOVA $(\mathrm{p}<0.010)$ showed 
258 that $8 \%$ of the total variance was assigned to between population diversity $(\mathrm{df}=11$, sum of

259 squares $=141.25$, variance components $=0.50)$ while $92 \%$ was assigned to diversity within

260 populations $(\mathrm{df}=167$, sum of squares $=952.44$, variance components $=5.70)$. As the county

261 structure did not explain much of the variation found in the microsatellite data, we clustered the

262 samples with STRUCTURE. The optimal number for $K$ was assessed both by the maximum log-

263 likelihood, which reached a plateau between $K=3$ and $K=5$, and by Delta $K$, which showed a

264 clear spike at $K=3$ (Figure 4a for Delta $K$ ). These three populations were not geographically

265 clear cut, although there was a 'northern' (Yorkshire, Cambridgeshire), a 'western' (Dorset,

266 some of the Shropshire and Welsh border populations, Wiltshire) and 'central/eastern'

267 (Leicester, Oxford, Sussex, Worcester) cluster. The remaining populations (Berkshire,

268 Hampshire, Kent) were admixed in approximately equal proportions of the 'eastern' and

269 'central/eastern' clusters. At $K=4$, the 'central/eastern' cluster forms two clusters and some of

270 the admixed populations become less admixed. At $K=5$, the 'central/eastern' cluster breaks

271 down even further but the populations are less resolved (Figure 4b).

272

273 Geographical distance

274 A Mantel test found no association between $\Phi_{\text {st }}$ (based on mtDNA results) and the geographical 275 distance between the populations $(\mathrm{r}=0.171, \mathrm{p}=0.120, \mathrm{n}=91)$. To examine whether variance

276 increased over geographical distance, the residuals from a linear regression of $\Phi_{\text {st }}$ against 277 geographical distance were plotted against geographical distance (Hutchison \& Templeton 278 1999); a Mantel test found no significant relationship $(\mathrm{r}=0.0001, \mathrm{p}=0.482, \mathrm{n}=91)$. Similarly, 279 the microsatellite DNA results showed no difference between $\mathrm{F}_{\mathrm{ST}}$ and the geographical distance $280 \quad(\mathrm{r}=0.215, \mathrm{n}=91, \mathrm{p}=0.150)$ 


\section{VKOR analysis}

283 A result could not be obtained for two rats (Kent $n=1$, Sussex $n=1)$, leaving a sample size of 284 184; VKORC1 variants were identified in $124(67.4 \%)$ individuals (Table 5). The proportion of 285 rats with VKORC1 variants varied between counties (Pearson's Chi-squared test: $X^{2}=29.59,13$ $286 \mathrm{df}, \mathrm{p}=0.005$ ) and the difference was apparently not due to sampling effort (Spearman's rank 287 correlation: $\mathrm{r}=-0.4, \mathrm{n}=14, \mathrm{p}=0.155)$. The Leu128Gln variant was not found in any of our 288 samples (the assay was checked using a positive control). Two VKORC1 variants were 289 identified in seventeen individuals (Tyr139Cys + Leu120Gln, n=12; Tyr139Cys + Tyr139Ser, n $290=4$; Leu120Gln + Tyr139Ser, $\mathrm{n}=1$ ); three variants were identified in a single individual (from 291 Hampshire); Tyr139Cys + Leu120Gln + Tyr139Ser. There was an association between 292 VKORC1 variant present and county $(\mathrm{G}=46.5$, df $=26, \mathrm{p}<0.01)$ but there was no association 293 between variant present and the population structure suggested by the microsatellite data $(\mathrm{G}=$ 294 14.9, df=12. N.S.).

296 A subsample of the rats (79) had been previously tested for susceptibility to warfarin by the

297 blood clotting response (BCR) test (Kerins et al. 2001); four were found to be susceptible. 298 However, in these four individuals the Tyr139Cys variant was identified; three of these 299 individuals were from one farm in Berkshire, the fourth, in which the Leu120Gln variant was 300 also identified, was from Gloucestershire. Furthermore, 10 of the 75 (BCR) resistant rats showed 301 none of the four VKORC1 variants that we had initially screened for. We sequenced exon 3 of 302 these $10 \mathrm{BCR}$ positive rats; in six we found no VKORC1 variants, in two of the remaining four 
303 we found the Leu120Gln variant, and in the other two we found a Tyr139Phe amino acid 304 substitution. 


\section{DISCUSSION}

307 We analysed genetic variation in rural brown rat populations in England using both

308 mitochondrial and microsatellite DNA in order to investigate the population structure of this

309 important pest species. In these populations we also quantified the number and type of VKORC1

310 variants previously reported to be associated with resistance to warfarin in the UK.

312 For the mtDNA data, we found two haplogroups, RNH1 and RNH6, and RNH2, RNH4, RNH5

313 and RNH3. The divergence between these two haplogroups was quite high (six mutational

314 positions) relative to the within group divergence (one-two mutational positions), suggesting that

315 these two clusters represent distinct founding haplotypes rather than in situ divergence. There is

316 no geographic structuring to the distribution of these haplogroups, suggesting either that they

317 were introduced and spread concurrently, or that they were introduced in two stages but

318 subsequently spread panmictically. This lack of mtDNA geographic structuring contrasts to that

319 found in another rodent pest, the house mouse, which has geographic structuring in the UK

320 (Searle et al. 2009), although this was detected at a wider scale than was found here.

321

322 In contrast, the STRUCTURE analysis of the microsatellite data suggested that there was some

323 degree of geographic population structuring within the UK, with a best fit of three populations

$324(K=3)$. There is some geographic coherence to the distribution of these populations, indicating

325 they are biologically relevant. A confounding variable is that there may also be a temporal 326 element, as the Leicester, Cambridgeshire and Yorkshire samples were all collected in 2005

327 rather than 1993-1994; Cambridgeshire and Yorkshire form a distinct cluster. To compare the 
328 results again to house mice, studies in Ireland and France found the microsatellite data strongly

329 matched the geographic origin of the samples (Jones et al. 2011).

330

331 Discrepancies of this kind between mitochondrial and microsatellite data have been found in

332 numerous studies (Waits et al. 2000). These discrepancies can be attributed to differences in the

333 levels of male and female gene flow (Avise 1994), as the dispersal distance of male rats is

334 greater than females (Calhoun 1962). However, this would imply a greater degree of geographic

335 structure shown by the (female dispersed) mtDNA data than that shown by the (male and female

336 dispersed) microsatellite data. A more likely explanation for these results is that the relatively

337 high mutation rate of microsatellites better reflects population structuring than the slower

338 mutating mtDNA sequence, which is informative about earlier events. This is particularly likely

339 to be the case as the fragment of mtDNA extracted here is relatively short.

340 The haplotype and nucleotide diversity of mtDNA in our samples is relatively low, although it is

341 similar to the values found for the Black rat on Madagascar (Hingston et al. 2005). We found

342 only six haplotypes differing at 10 positions. Low nucleotide diversity found in a widespread

343 species is often attributed to a slow range expansion following a small population size (founder/

344 bottleneck effects) (Joseph et al. 2002). Further support for this is given by the

345 distribution of pairwise haplotype differences (Figure 3), which matches that expected under a

346 spatial expansion model (Rogers \& Harpending 1992).

348 If, as seems likely from our data, the English brown rat population has undergone a recent (on an

349 evolutionary scale) expansion, it is unlikely to be yet at equilibrium. Regional equilibrium can be

350 tested by comparing $\mathrm{F}_{\text {st }}$ to geographical distance between regions (Hutchison \& Templeton 
351 1999). If the population has reached equilibrium, there will be a linear relationship between $\mathrm{F}_{\text {st }}$

352 and geographical distance. We found no significant relationship between $\Phi_{\mathrm{st}}$ (analogous to $\mathrm{F}_{\mathrm{st}}$ )

353 and geographical distance. Our results most closely resemble Hutchison \& Templeton's case III,

354 where the population is fragmented into small, isolated populations and drift becomes more

355 important than gene flow. This allows allele frequencies in each population to drift

356 independently relative to geographical distance and random sampling of gametes creates a large

357 degree of variance between the plotted points (Hutchison \& Templeton 1999). We found no

358 significant correlation between the residual of $\Phi_{\text {st }}$ and geographical distance (a measure of the

359 degree of variance) and geographical distance, indicating that our data do indeed fit the case III

360 model. This model and our data suggest that the English rural rat population is not yet at

361 equilibrium and that gene flow is less important than drift in explaining the genetic structure

362 found.

363

364 Accordingly, we found a geographical trend (by county) in the distribution of VKORC1 variants.

365 The Leu120Gln amino acid substitution was found in the central and southern counties. The

366 Tyr139Cys substitution was the most common and found in the majority in almost all counties;

367 in Hampshire a combination of Tyr139Cys and Leu120Gln substitutions was found more

368 frequently than Tyr139Cys alone, although the sample size for the county (nine) was relatively

369 small. The Tyr139Cys substitution is reportedly better at ameliorating the effects of (and

370 therefore more likely to confer a selective advantage against) warfarin use than Leu120Gln (Pelz

371 et al. 2005), which potentially provides an explanation for the more widespread distribution of

372 Tyr139Cys. The Tyr139Cys amino acid substitution is almost ubiquitous in Germany and

373 Denmark (Pelz et al. 2005). We found only five instances of the Tyr139Ser variant; all of these 
374 were in combination with other VKORC1 amino acid substitutions. The Tyr139Ser variant has

375 previously been reported from rats near the Anglo-Welsh borders around the town of Welshpool,

376 and not from elsewhere in the UK (Buckle 2013). Our two records of this VKORC1 variant from

377 Shropshire were from rats collected near the town of Shrewsbury, 30km from Welshpool. Our

378 two records of this variant from Yorkshire, and the single record from Hampshire are noteworthy

379 given their considerable distance from previous records, although it is possible that these

380 represent false positives; Mln I digestion of the Tyr139Ser variant may produce fragments close

381 in size (110 bp plus $<50 \mathrm{bp})$ to wild type alleles (160 bp plus $<50 \mathrm{bp}$ ) (Pelz et al. 2005).

382

383 We found a mis-match between BCR results and VKOR polymorphisms for some rats;

384 Tyr139Cys amino acid substitution was identified in four apparently susceptible rats. Pelz et al.

385 (2005) suggested that such cases were false negatives due to inaccuracies of the BCR test,

386 although an alternative explanation is that the Tyr139Cys amino acid substitution alone is not

387 sufficient to confer resistance, and an additional (undetected) substitution (or other physiological

388 mechanism) is required which these four rats did not possess. We also found that six BCR

389 positive rats had no VKORC1 amino acid substitutions, that is, they were BCR false positives,

390 unless there is an as yet undiscovered, alternative resistance mechanism (Pelz et al. 2005), and a

391 similar result has been reported elsewhere (Heiberg 2009). The Leu120Gln variant was identified

392 in two BCR positive rats that were initially thought to be false positives, whilst in a further two

393 apparent BCR false positives, a Tyr139Phe amino acid substitution not known from the UK at

394 the time of our study was found, although this VKORC1 variant has since been reported in rats

395 from Kent (Prescott et al. 2010). Several other newly-discovered VKORC1 variants have

396 recently been reported from the UK (Buckle 2013). We initially screened samples for the four 
397 VKORC1 variants reported from the UK at the time of our study, and did not look for other

398 variants, except where BCR false positives were suspected. It is possible therefore that we

399 underestimated the prevalence of VKOR polymorphisms in our samples. However, the more

400 recently reported VKORC1 variants also appear to have limited (regional) distribution, which is

401 consistent with our suggestion that limited gene flow between regions limits geographical

402 distribution of VKOR polymorphisms.

403

404 It has been reported that because of pleiotropic costs (on vitamin $\mathrm{K}$ requirements, reduced

405 fecundity and growth rate) that anticoagulant resistant rats (from some regions) are at a selective

406 disadvantage in the absence of anticoagulant use compared to susceptible individuals (Jacob et

407 al. 2012; Smith et al. 1991). Intuitively therefore, these susceptible rats should out-compete their

408 resistant counterparts in the absence of exposure to anticoagulant rodenticides, and it follows that

409 resistance-management strategies should, where possible, include the use of non-anticoagulant

410 rodenticides and non-rodenticide approaches in order to remove selection pressure, and to

411 remove resistant individuals (Buckle 2013; Greaves 1995; Lambert et al. 2008; Quy et al. 1995;

412 Smith \& Greaves 1987). However, in a captive, insular rat population, anticoagulant tolerance

413 was not significantly influenced in the absence of bromadiolone selection (Heiberg et al. 2006),

414 and not all warfarin resistant strains appear to be at a selective disadvantage in the absence of

415 poison use (Smith et al. 1993). In a field trial where removal of anticoagulant selection pressure

416 from a population of rats highly resistant to bromadiolone did not result in a greatly increased

417 proportion of susceptible individuals within the population, it was found that a high proportion of

418 rats on surrounding farmsteads were also resistant to bromadiolone, and there was therefore little

419 opportunity for susceptible rats to dilute the resistant population through immigration (CSL 
420 2002). The lack of mixing between populations at larger geographical scales, revealed by this

421 study, is likely to intensify the effect of local selection pressure imposed by sustained

422 anticoagulant use, and reversing these processes is therefore likely to be slow and difficult to

423 achieve. However, the present study also suggests that restricted gene flow between rat

424 populations should limit the rate of spread of resistant populations to some degree, and should

425 therefore make their targeted management a realistic possibility. Greaves (1995) suggested that

426 prompt and sustained control programmes using a suitable range of non-selective techniques

427 within a $20 \mathrm{~km}$ radius of anticoagulant foci would be very likely to extinguish the majority of

428 resistant populations. However, Buckle (2013) noted that such large-scale coordinated resistance

429 management efforts have previously proven prohibitively expensive or impractical in the UK.

430 Some of the most toxic second-generation anticoagulant rodenticides (SGARs) including

431 brodifacoum and flocoumafen are still effective against rats resistant to warfarin, bromadiolone

432 and difenacoum, and their use in areas where resistance to the less-toxic anticoagulants is

433 encountered has been recommended where alternative (non-anticoagulant) methods cannot be

434 used (Buckle 2013). However, it is unknown what the impacts of this approach will be on

435 resistant rat populations in the longer-term, and it remains important that alternative rodent

436 control approaches and rodenticides are developed, so that a range of viable options is available

437 to manage populations of anticoagulant resistant rats. 


\section{Acknowledgements}

439 We thank Carl Smith for his involvement with the work, and two anonymous referees who 440 provided useful feedback on an earlier draft. We are grateful to Geoff Butcher from the 441 Babraham Institute for providing the additional samples from Cambridge.

442 
443

444

445

446

447

448

449

450

451

452

453

454

455

456

457

458

459

460

461

462

463

464

465

466

467

468

469

470

471

472

473

474

475

476

477

478

479

480

481

482

483

484

485

486

487

\section{References}

Avise JC. 1994. Molecular markers, natural history and evolution. New York: Chapman \& Hall. Balloux F, and Lugon-Moulin N. 2002. The estimation of population differentiation with microsatellite markers. Molecular Ecology 11:155-165.

Bell RG, and Caldwell PT. 1973. Mechanisms of warfarin resistance. Biochemistry 12:17591762.

Boyle CM. 1960. Case of apparent resistance of Rattus norvegicus Berkenhout to anticoagulant poisons. Nature 188:517.

Brodie J. 1976. Anticoagulant resistant rats (Rattus norvegicus) in Scotland. International pest control 18:7-10.

Buckle A. 2013. Anticoagulant resistance in the United Kingdom and a new guideline for the management of resistant infestations of Norway rats (Rattus norvegicus Berk.). Pest Management Science 69:334-341.

Buckle A, and Smith R. 2015. Rodent pests and their control: $2^{\text {nd }}$ Edition. Oxford: CABI.

Calhoun JB. 1962. The ecology and sociology of the Norway rat. U.S. Department of Health, Education and welfare. $\mathrm{p} 288$.

CSL. 2002. Development of guidelines on best practice for rodenticide use. Report to the Department of Environment, Food and Rural Affairs.

http://randd.defra.gov.uk/Document.aspx?Document=PV1016 917 FRP.doc.

Drummond DC. 1970. Variation in rodent populations in response to control measures. Symposia of the Zoological Society of London 26:351-367.

Earl DA, and vonHoldt BM. 2012. STRUCTURE HARVESTER: a website and program for visualizing STRUCTURE output and implementing the Evanno method. Conservation genetics resources 4:359-361.

Evanno G, Regnaut S, and Goudet J. 2005. Detecting the number of clusters of individuals using the software STRUCTURE: a simulation study. Molecular Ecology 14:2611-2620.

Excoffier L, Laval G, and Schneider S. 2005. ARLEQUIN ver. 3.0: An integrated software package for population genetics data analysis. Evolutionary Bioinformatics Online 1:4750 .

Greaves JH. 1995. Managing resistance to anticoagulant rodenticides: An appraisal. Pesticide science 43:79-82.

Greaves JH, and Ayres PB. 1967. Heritable resistance to warfarin in rats. Nature 215:877-878.

Hadler M, and Shadbolt R. 1975. Novel 4-hydroxycoumarin anticoagulants active against resistant rats. Nature 253:275.

Hadler MR, Redfern R, and Rowe FP. 1975. Laboratory evaluation of difenacoum as a rodenticide. Journal of Hygiene, Cambridge 74:441-448.

Hayes WJ. 1950. Control of Norway rats with residual rodenticide warfarin. Public Health Reports 65:1537-1555.

Heiberg A-C. 2002. Population effects of anticoagulant rodenticide resistance in Norway Rats (Rattus norvegicus). PhD Thesis. University of Copenhagen.

Heiberg AC. 2009. Anticoagulant resistance: a relevant issue in sewer rat (Rattus norvegicus) control? Pest Management Science 65:444-449.

Heiberg AC, Leirs H, and Siegismund HR. 2006. Reproductive success of bromadiolone-resistant rats in absence of anticoagulant pressure. Pest Management Science 62:862-871. 
488

489

490

491

492

493

494

495

496

497

498

499

500

501

502

503

504

505

506

507

508

509

510

511

512

513

514

515

516

517

518

519

520

521

522

523

524

525

526

527

528

529

530

531

532

Hingston M, Goodman SM, Ganzhorn JU, and Sommer S. 2005. Reconstruction of the colonization of southern Madagascar by introduced Rattus rattus. Journal of Biogeography 32:1549-1559.

Huson DH. 1998. SplitsTree: analyzing and visualizing evolutionary data. Bioinformatics 14:6873.

Hutchison DW, and Templeton AR. 1999. Correlation of pairwise genetic and geographic distance measures: Inferring the relative influences of gene flow and drift on the distribution of genetic variability. Evolution 53:1898-1914.

Jacob J, Endepols S, Pelz HJ, Kampling E, Cooper TG, Yeung CH, Redmann K, and Schlatt S. 2012. Vitamin $\mathrm{K}$ requirement and reproduction in bromadiolone-resistant Norway rats. Pest Management Science 68:378-385.

Jakobsson M, and Rosenberg NA. 2007. CLUMPP: a cluster matching and permutation program for dealing with label switching and multimodality in analysis of population structure. Bioinformatics 23:1801-1806.

Jones EP, Jóhannesdóttir F, Gündüz I, Richards MB, and Searle J. 2011. The expansion of the house mouse into north-western Europe. Journal of Zoology 283:257-268.

Joseph L, Wilke T, and Alpers D. 2002. Reconciling genetic expectations from host specificity with historical population dynamics in an avian brood parasite, Horsfield's BronzeCuckoo Chalcites basalis of Australia. Molecular Ecology 11:829-837.

Kerins G, Dennis N, Atterby H, Gill J, and MacNicoll A. 2001. Distribution of resistance to anticoagulant rodenticides in the Norway rat (Rattus norvegicus Berk.) in England 199598. Advances in Vertebrate Pest Management II 2:149-159.

Lambert MS, Quy RJ, Smith RH, and Cowan DP. 2008. The effect of habitat management on home-range size and survival of rural Norway rat populations. Journal of Applied Ecology 45:1753-1761.

Lund M. 1985. The "second generation" anticoagulants: A review. Acta Zoologica Fennica 173:149-153.

Meehan AP. 1984. Rats and mice: Their biology and control: Rentokil Ltd.

Peakall R, and Smouse PE. 2006. GENALEX 6: genetic analysis in Excel. Population genetic software for teaching and research. Molecular Ecology Notes 6:288-295.

Pelz HJ, Rost S, Hunerberg M, Fregin A, Heiberg AC, Baert K, MacNicoll AD, Prescott CV, Walker AS, Oldenburg J, and Muller CR. 2005. The genetic basis of resistance to anticoagulants in rodents. Genetics 170:1839-1847.

Prescott CV, Buckle AP, Gibbings JG, Allan EN, and Stuart AM. 2010. Anticoagulant resistance in Norway rats (Rattus norvegicus Berk.) in Kent-a VKORC1 single nucleotide polymorphism, tyrosine139phenylalanine, new to the UK. International Journal of Pest Management 57:61-65.

Pritchard JK, Stephens M, and Donnelly P. 2000. Inference of population structure using multilocus genotype data. Genetics 155:945-959.

Quy RJ, Cowan DP, Prescott CV, Gill JE, Kerins GM, Dunsford G, Jones A, and Macnicoll AD. 1995. Control of a population of Norway rats resistant to anticoagulant rodenticides. Pesticide science 45:247-256.

Redfern R, and Gill JE. 1978. The development and use of a test to identify resistance to the anticoagulant difenacoum in the Norway rat (Rattus norvegicus). Journal of Hygiene $81: 427-431$. 
533 Rogers AR, and Harpending H. 1992. Population growth makes waves in the distribution of

534

535

536

537

538

539

540

541

542

543

544

545

546

547

548

549

550

551

552

553

554

555

556

557

558

559

560

561

562

563

564

565

566

567

568

569

570

571

572 pairwise genetic differences. Molecular Biology and Evolution 9:552-569.

Rosenberg NA. 2004. DISTRUCT: a program for the graphical display of population structure. Molecular Ecology Notes 4:137-138.

Rost S, Fregin A, Ivaskevicius V, Conzelmann E, Hörtnagel K, Pelz H-J, Lappegard K, Seifried E, Scharrer I, and Tuddenham EG. 2004. Mutations in VKORC1 cause warfarin resistance and multiple coagulation factor deficiency type 2. Nature 427:537-541.

Roush RT, and McKenzie JA. 1987. Ecological genetics of insecticide and acaricide resistance. Annual Review of Entomology 32:361-380.

Sadowski JA, Esmon CT, and Suttie JW. 1976. Vitamin K-dependent carboxylase. Requirements of the rat liver microsomal enzyme system. Journal of Biological Chemistry 251:27702776.

Searle JB, Jones CS, Gündüz İ, Scascitelli M, Jones EP, Herman JS, Rambau RV, Noble LR, Berry R, and Giménez MD. 2009. Of mice and (Viking?) men: phylogeography of British and Irish house mice. Proceedings of the Royal Society B: Biological Sciences 276:201207.

Smith P, Berdoy M, Smith RH, and Macdonald DW. 1993. A new aspect of warfarin resistance in wild rats: benefits in the absence of poison. Functional Ecology 7:190-194.

Smith P, Townsend MG, and Smith RH. 1991. A cost of resistance in the brown rat? Reduced growth rate in warfarin-resistant lines. Functional Ecology 5:441-447.

Smith RH, and Greaves JH. 1987. Resistance to anticoagulant rodenticides: the problem and its management. In: Donahaye E, and Navarro S, editors. Proceedings of the $4^{\text {th }}$ International Working Conference on Stored-Product Protection. Agricultural Research Organisation, Bet Dagan. p 302-315.

Suttie JW. 1980. Mechanism of action of vitamin K: synthesis of gamma-carboxyglutamic acid. CRC Critical Reviews in Biochemistry 8:191-223.

Tamura K, and Nei M. 1993. Estimation of the number of nucleotide substitutions in the control region of mitochondrial DNA in humans and chimpanzees. Molecular Biology and Evolution 10:512-526.

Waits L, Taberlet P, Swenson JE, Sandegren F, and Franzen R. 2000. Nuclear DNA microsatellite analysis of genetic diversity and gene flow in the Scandinavian brown bear (Ursus arctos). Molecular Ecology 9:421-431.

Webster JP, Ellis WA, and MacDonald DW. 1995. Prevalence of Leptospira spp. in wild brown rats ( Rattus norvegicus ) on UK farms. Epidemiology and Infection 114:195-201.

Wool D, and Noiman S. 1983. Integrated control of insecticide resistance by combined genetic and chemical treatments: a warehouse model with flour beetles (Tribolium; Tenebrionidae, Coleoptera). Zeitschrift für Angewandte Entomologie 95:22-30.

Wright S. 1951. The genetical structure of populations. Annals of Eugenics 15.

Yalden D. 1990. The History of British Mammals. Cambridge: Cambridge University Press. 


\section{Table $\mathbf{1}$ (on next page)}

Variations in exon 3 of VKORC1 (after Pelz et al. 2005).

The variations are labeled as wildtype/position/mutant, so Tyr139Cys is a tyrosine at the 139th amino acid converted to a cysteine in the variant. 
$1 \quad$ Table 1

2

\begin{tabular}{l|lll}
\hline Mutation & Detection method & Wildtype & Mutant \\
& & & \\
\hline Tyr139Cys & ARMS-PCR & $168 \mathrm{bp}$ & $168 \mathrm{bp}$ \\
& & $123 \mathrm{bp}$ & $101 \mathrm{bp}$ \\
Leu120Gln & Stu I & $330 \mathrm{bp}$ & $195 \mathrm{bp}$ \\
& & & $135 \mathrm{bp}$ \\
Leu128Gln & Bsr I & $330 \mathrm{bp}$ & $170 \mathrm{bp}$ \\
& & & $160 \mathrm{bp}$ \\
Tyr139Ser & Mnl I & $160 \mathrm{bp}$ & $110 \mathrm{bp}$ \\
\hline
\end{tabular}

4 


\section{Table 2 (on next page)}

The nucleotide differences of the 6 mtDNA haplotypes (RNH1-RNH6) in a sample of 185 Rattus norvegicus individuals from 15 different sampling areas in the United Kingdom.

The top row is the position of the variable nucleotides within the $425 \mathrm{bp}$ sequence. 
$1 \quad$ Table 2

2

\begin{tabular}{|c|c|c|c|c|c|c|c|c|c|c|c|}
\hline Position & 95 & 97 & 157 & 204 & 244 & 246 & 260 & 265 & 276 & 313 & $n$ \\
\hline \multicolumn{12}{|l|}{ Haplotype } \\
\hline RNH1 & $\mathrm{T}$ & $\mathrm{T}$ & $\mathrm{C}$ & $\mathrm{T}$ & $\mathrm{C}$ & $\mathrm{T}$ & $\mathrm{T}$ & $\mathrm{G}$ & A & $\mathrm{G}$ & 130 \\
\hline RNH2 & . & $\mathrm{C}$ & . & $\mathrm{C}$ & . & $\mathrm{C}$ & $\mathrm{C}$ & A & $\mathrm{G}$ & A & 49 \\
\hline RNH3 & . & $\mathrm{C}$ & $\mathrm{T}$ & $\mathrm{C}$ & $\mathrm{T}$ & $\mathrm{C}$ & . & A & $\mathrm{G}$ & A & 3 \\
\hline RNH4 & $\mathrm{C}$ & $\mathrm{C}$ & . & $\mathrm{C}$ & . & $\mathrm{C}$ & $\mathrm{C}$ & A & $\mathrm{G}$ & A & 1 \\
\hline RNH5 & & $\mathrm{C}$ & . & $\mathrm{C}$ & . & $\mathrm{C}$ & . & A & G & A & 1 \\
\hline RNH6 & & . & . & . & . & . & . & . & . & A & 1 \\
\hline
\end{tabular}

3

4 


\section{Table 3(on next page)}

Haplotype $(h)$ and nucleotide diversity $(\pi)$ and their standard deviations (SD) based on mtDNA of the populations sampled. 
$1 \quad$ Table 3

2

\begin{tabular}{|c|c|c|c|}
\hline Area & $n$ & $h \pm S D$ & $\pi \pm S D$ \\
\hline Total & 185 & $0.44 \pm 0.03$ & $0.0069 \pm 0.0040$ \\
\hline Berkshire & 25 & $0.49 \pm 0.09$ & $0.0077 \pm 0.0046$ \\
\hline Cambridgeshire & 21 & $0.27 \pm 0.12$ & $0.0043 \pm 0.0029$ \\
\hline Dorset & 7 & $0.00 \pm 0.00$ & $0.0000 \pm 0.0000$ \\
\hline Gloucestershire & 3 & $0.67 \pm 0.31$ & $0.0118 \pm 0.0098$ \\
\hline Greater London & 3 & $0.00 \pm 0.00$ & $0.0000 \pm 0.0000$ \\
\hline Hampshire & 9 & $0.00 \pm 0.00$ & $0.0000 \pm 0.0000$ \\
\hline Kent & 7 & $0.57 \pm 0.12$ & $0.0101 \pm 0.0065$ \\
\hline Leicestershire & 35 & $0.00 \pm 0.00$ & $0.0000 \pm 0.0000$ \\
\hline North Yorkshire & 24 & $0.51 \pm 0.05$ & $0.0089 \pm 0.0052$ \\
\hline Oxfordshire & 10 & $0.53 \pm 0.10$ & $0.0094 \pm 0.0058$ \\
\hline Shropshire + Powys & 6 & $0.60 \pm 0.21$ & $0.0123 \pm 0.0085$ \\
\hline Sussex & 12 & $0.41 \pm 0.13$ & $0.0072 \pm 0.0045$ \\
\hline Wiltshire & 18 & $0.31 \pm 0.13$ & $0.0049 \pm 0.0032$ \\
\hline Worcestershire & 5 & $0.40 \pm 0.24$ & $0.0071 \pm 0.0052$ \\
\hline
\end{tabular}




\section{Table 4(on next page)}

Microsatellite alleles. 
$1 \quad$ Table 4

\begin{tabular}{|c|c|c|c|c|c|c|c|c|c|c|c|c|c|}
\hline \multirow[b]{2}{*}{ County } & \multicolumn{6}{|c|}{ Number of Alleles in each Locus } & \multirow{2}{*}{$\begin{array}{l}\text { Mean } \\
\text { number } \\
\text { of alleles }\end{array}$} & \multicolumn{5}{|c|}{ Heterozygosity (Observed/Expected) } & \multirow[b]{2}{*}{ D8 } \\
\hline & D17 & D3 & D5 & D12 & D14 & D8 & & D17 & D3 & D5 & D12 & D14 & \\
\hline Berkshire & 8 & 9 & 5 & 15 & 9 & 11 & 9.67 & $0.32 / 0.74$ & $0.76 / 0.83$ & $0.44 / 0.71$ & $0.84 / 0.90$ & $0.68 / 0.85$ & $0.60 / 0.90$ \\
\hline Cambridge & 5 & 8 & 6 & 12 & 6 & 11 & 8.00 & $0.38 / 0.36$ & $0.85 / 0.80$ & $0.76 / 0.72$ & $0.76 / 0.85$ & $0.62 / 0.70$ & $0.71 / 0.83$ \\
\hline Dorset & 6 & 7 & 5 & 5 & 5 & 5 & 5.50 & $0.71 / 0.83$ & $0.71 / 0.82$ & $0.85 / 0.80$ & $0.43 / 0.77$ & $0.57 / 0.86$ & $1.00 / 0.78$ \\
\hline Hampshire & 6 & 5 & 2 & 7 & 6 & 5 & 5.16 & $0.77 / 0.84$ & $0.77 / 0.78$ & $0.44 / 0.50$ & $0.66 / 0.83$ & $0.66 / 0.80$ & $0.55 / 0.67$ \\
\hline Kent & 8 & 6 & 2 & 9 & 7 & 7 & 6.50 & $0.75 / 0.85$ & $0.87 / 0.83$ & $0.50 / 0.40$ & $0.62 / 0.91$ & $0.87 / 0.89$ & $0.62 / 0.90$ \\
\hline Leicestershire & 9 & 8 & 6 & 10 & 11 & 12 & 9.33 & $0.31 / 0.70$ & $0.74 / 0.80$ & $0.60 / 0.64$ & $0.71 / 0.76$ & $0.83 / 0.87$ & $0.77 / 0.88$ \\
\hline North Yorkshire & 9 & 10 & 5 & 10 & 10 & 12 & 9.33 & $0.31 / 0.68$ & $0.79 / 0.82$ & $0.37 / 0.64$ & $0.79 / 0.86$ & $0.62 / 0.86$ & $0.79 / 0.90$ \\
\hline Oxfordshire & 7 & 6 & 2 & 9 & 8 & 8 & 6.67 & $0.50 / 0.83$ & $0.70 / 0.79$ & $0.40 / 0.48$ & $0.50 / 0.92$ & $0.70 / 0.88$ & $0.70 / 0.90$ \\
\hline Shropshire + Powys & 4 & 6 & 5 & 7 & 6 & 6 & 5.67 & $0.33 / 0.45$ & $0.83 / 0.86$ & $0.50 / 0.79$ & $0.50 / 0.83$ & $0.66 / 0.87$ & $0.50 / 0.86$ \\
\hline Sussex & 7 & 6 & 4 & 10 & 7 & 8 & 7.00 & $0.45 / 0.69$ & $0.72 / 0.85$ & $0.27 / 0.61$ & $0.72 / 0.90$ & $0.72 / 0.75$ & $0.63 / 0.87$ \\
\hline Wiltshire & 6 & 9 & 5 & 11 & 7 & 10 & 8.00 & $0.22 / 0.61$ & $0.72 / 0.78$ & $0.66 / 0.66$ & $0.83 / 0.78$ & $0.66 / 0.77$ & $0.61 / 0.81$ \\
\hline Worcester & 3 & 5 & 2 & 5 & 4 & 6 & 4.16 & $0.60 / 0.68$ & $1.00 / 0.82$ & $0.00 / 0.53$ & $0.80 / 0.87$ & $0.40 / 0.77$ & $0.20 / 0.91$ \\
\hline
\end{tabular}


Table 5 (on next page)

Distribution of VKORC1 variants by county. 
2

\begin{tabular}{l|cccccccc}
\hline & & No mutation & & & & Tyr139Cys, \\
County & $\mathrm{N}$ & $\begin{array}{c}\text { Tyr139Cys, } \\
\text { Leu120Gln }\end{array}$ & $\begin{array}{c}\text { Tyr139Cys, } \\
\text { Teu120Gln, } \\
\text { Tyr139Ser }\end{array}$ \\
\hline Berkshire & 25 & $3(12.0)$ & 16 & 1 & 0 & 5 & 0 & 0 \\
Cambridgeshire & 21 & $8(38.1)$ & 13 & 0 & 0 & 0 & 0 & 0 \\
Dorset & 7 & $3(42.9)$ & 4 & 0 & 0 & 0 & 0 & 0 \\
Gloucestershire & 3 & $0(0.0)$ & 2 & 0 & 0 & 1 & 0 & 0 \\
Greater London & 3 & $1(33.3)$ & 2 & 0 & 0 & 0 & 0 & 0 \\
Hampshire & 9 & $2(22.2)$ & 1 & 1 & 0 & 4 & 0 & 1 \\
Kent & 7 & $3(42.9)$ & 4 & 0 & 0 & 0 & 0 & 0 \\
Leicestershire & 35 & $19(54.3)$ & 16 & 0 & 0 & 0 & 0 & 0 \\
North Yorkshire & 24 & $13(54.2)$ & 8 & 1 & 0 & 0 & 2 & 0 \\
Oxfordshire & 10 & $2(20.0)$ & 7 & 0 & 0 & 1 & 0 & 0 \\
Powys & 2 & $1(50.0)$ & 1 & 0 & 0 & 0 & 0 & 0 \\
Shropshire & 4 & $0(0.0)$ & 2 & 0 & 0 & 0 & 2 & 0 \\
Sussex & 11 & $0(0.0)$ & 8 & 1 & 0 & 2 & 0 & 0 \\
Wiltshire & 18 & $5(27.8)$ & 11 & 2 & 0 & 0 & 0 & 0 \\
Worcestershire & 5 & $0(0.0)$ & 5 & 0 & 0 & 0 & 0 & 0 \\
\hline Total & 184 & $60(32.6)$ & 100 & 6 & 0 & 13 & 4 & 1 \\
\hline
\end{tabular}




\section{1}

Geographical distribution of samples.

Shading denotes (historical) English counties, Wales and Scotland Local Authorities are shown unshaded. Contains Ordnance Survey data (c) Crown Copyright 2015.

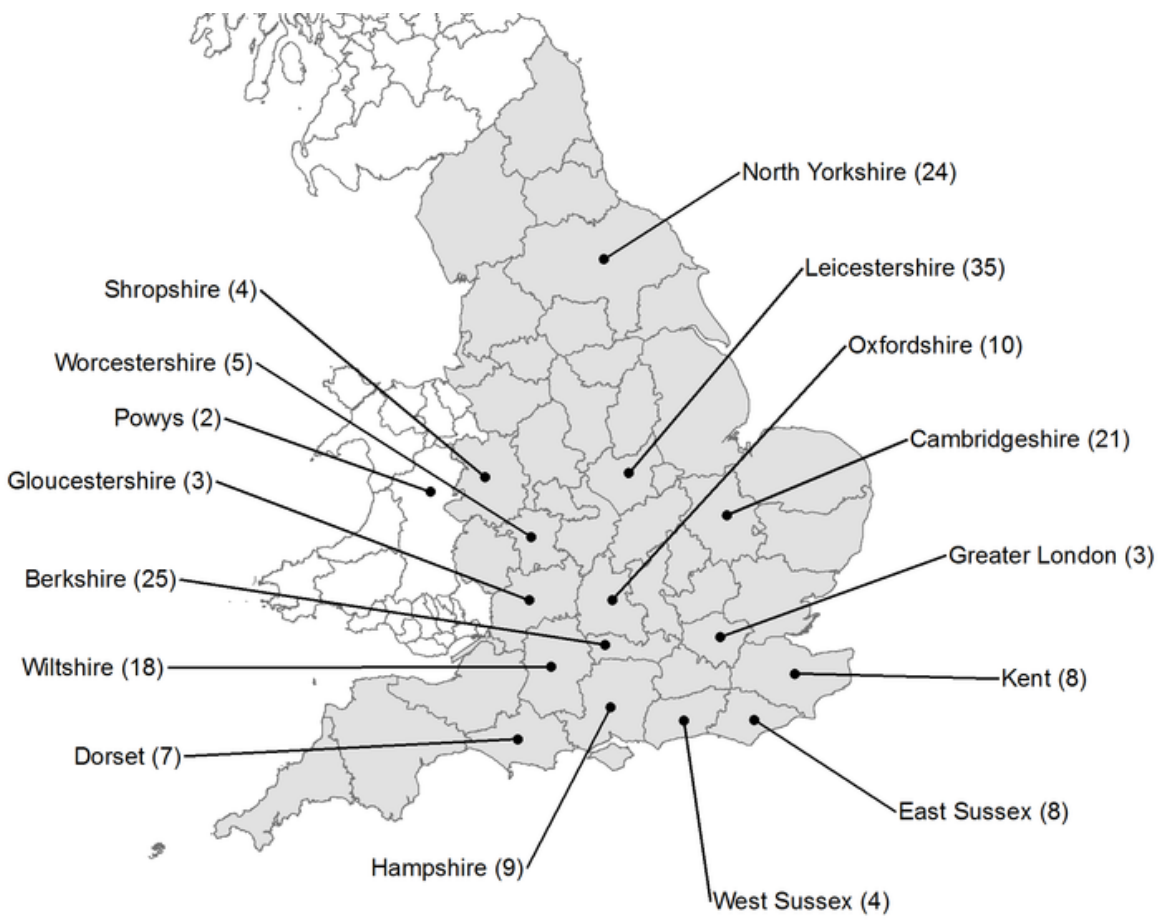


2

Phylogenetic relationships from mtDNA; study samples and closely-related Genbank sequences.

A) Minimum spanning network of the phylogenetic relationships between the 6 mtDNA haplotypes found. The area of the circles represents the frequency of the haplotypes in the entire population. B ) Neighbourjoining tree calculated with Tamura $\&$ Nei (1993) distances for the 6 mtDNA haplotypes of English brown rats (RNH1-6), several strains of lab rats (Wistar - Accession numbers:MIRNXX, RNMITDLO, Sprague-Dawley - MIRNDNC, BN/SsNHsdMCW - AY172581, F344 X BN F1 - AY769440), a Danish wild caught brown rat RN0428514 and the closely related black rat $R$. rattus - DQ009794. The percentage bootstrap support (1000 replicates) are shown for nodes with greater than $50 \%$ support. 
A)

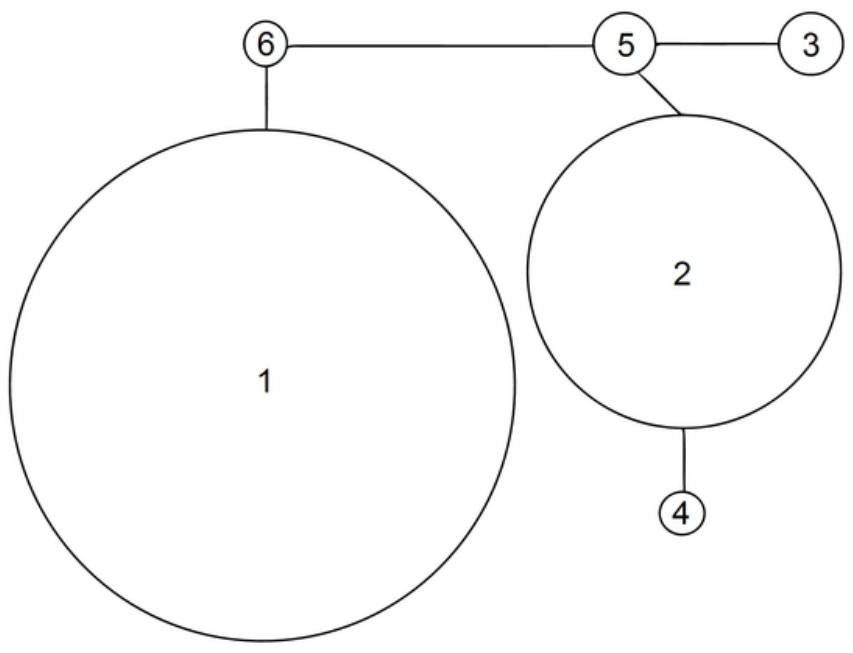

B)

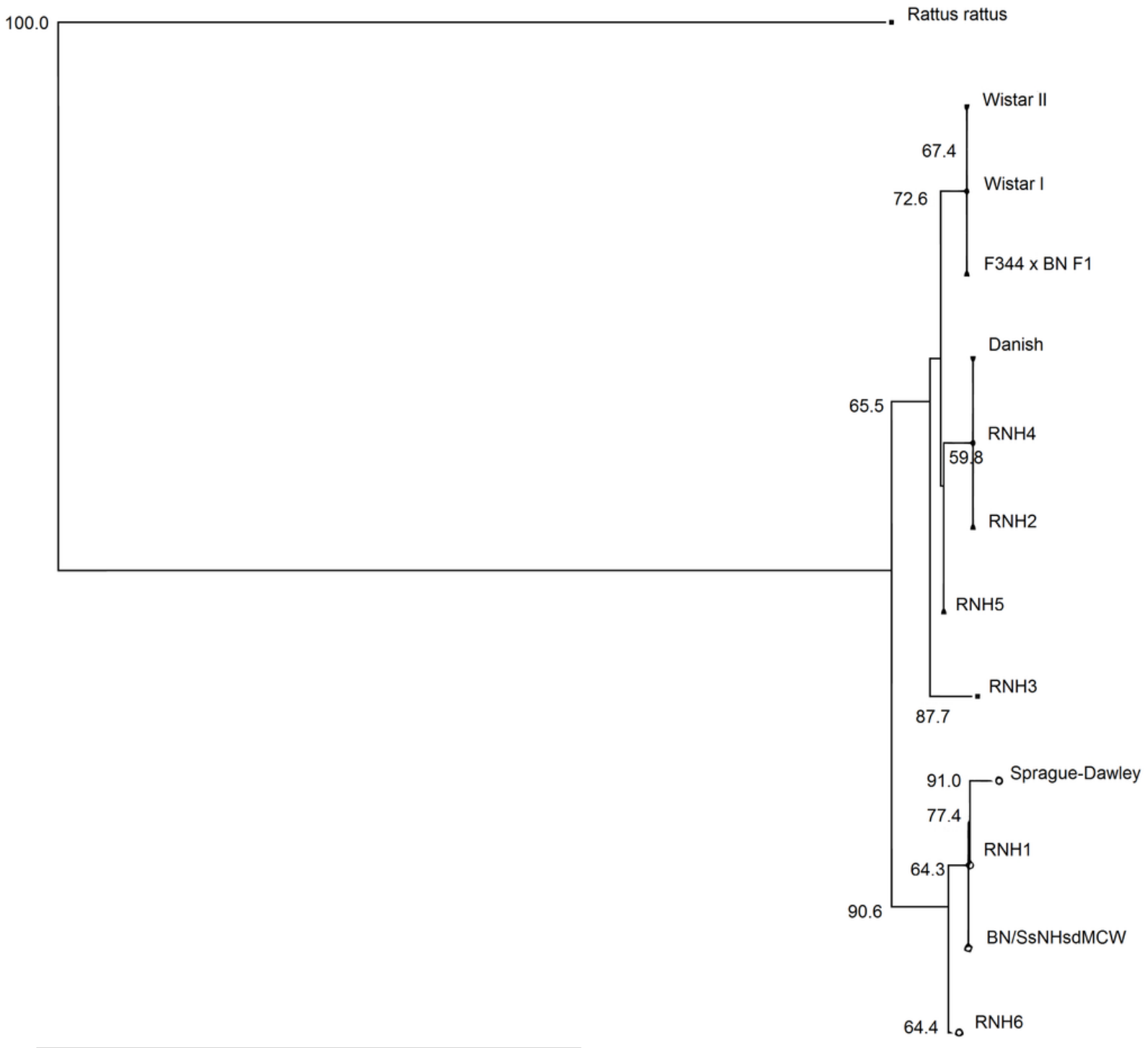


3

Mismatch distribution of pairwise sequence differences in brown rat mtDNA (bars).

The dotted line represents the expected results from a spatial expansion model.

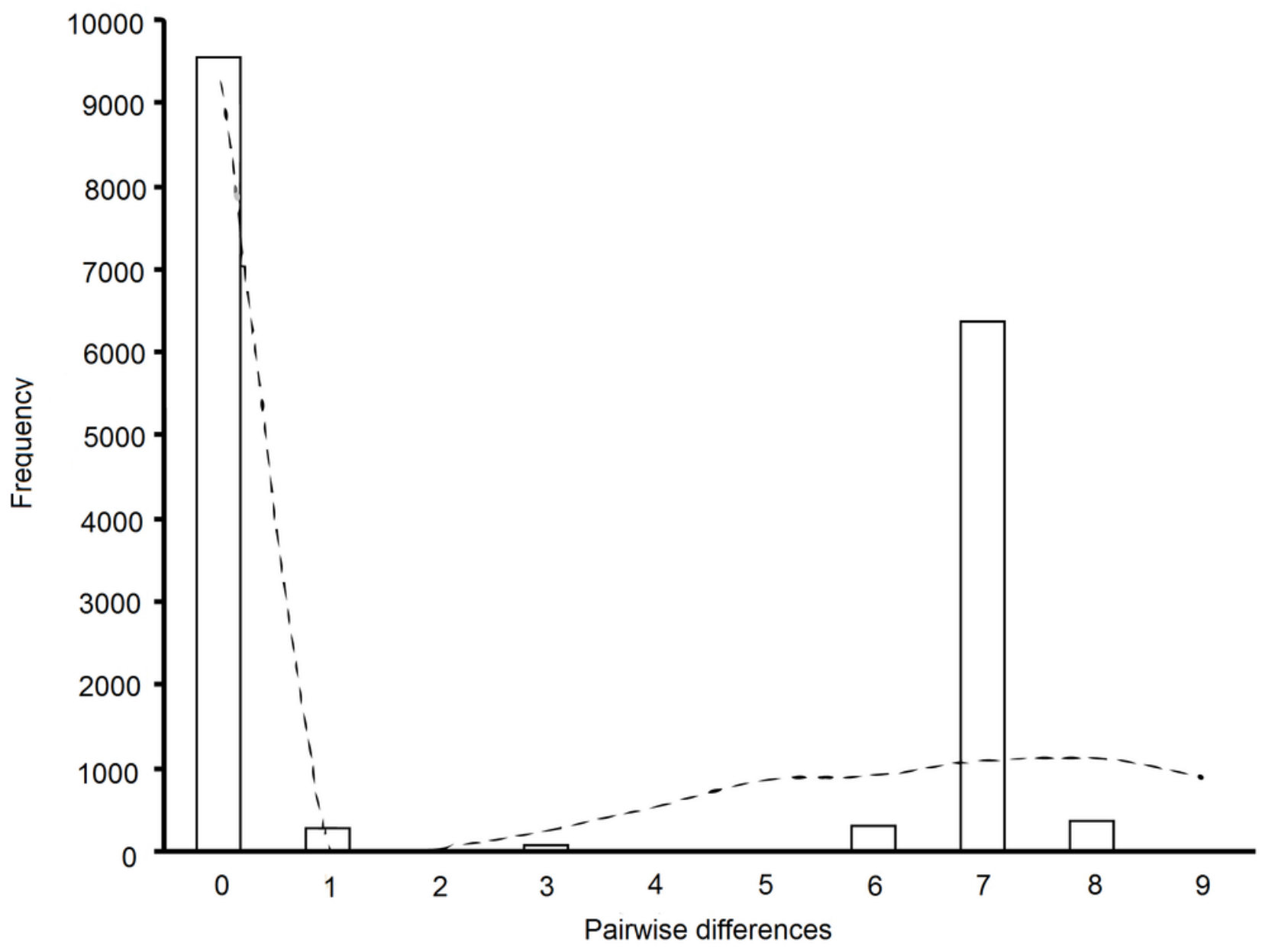




\section{4}

Population structure of brown rats from microsatellite DNA data.

A) shows the rate of change in the log-likelihood values between the number of genetic populations, $K$, for $K=2$ to $K=11$, showing that the value of $K$ with the greatest support is $K$ $=3$. B) STRUCURE plots of the average of ten runs of $K$ for $K=2, K=3$ and $K=4$, showing the allocation of each individual (a single vertical bar) to each population, grouped by geographic sampling location along the horizontal axis. Each geographic location is divided by a thin black line, and the abbreviated geographic location is given at the bottom of $K=4$. 
A.

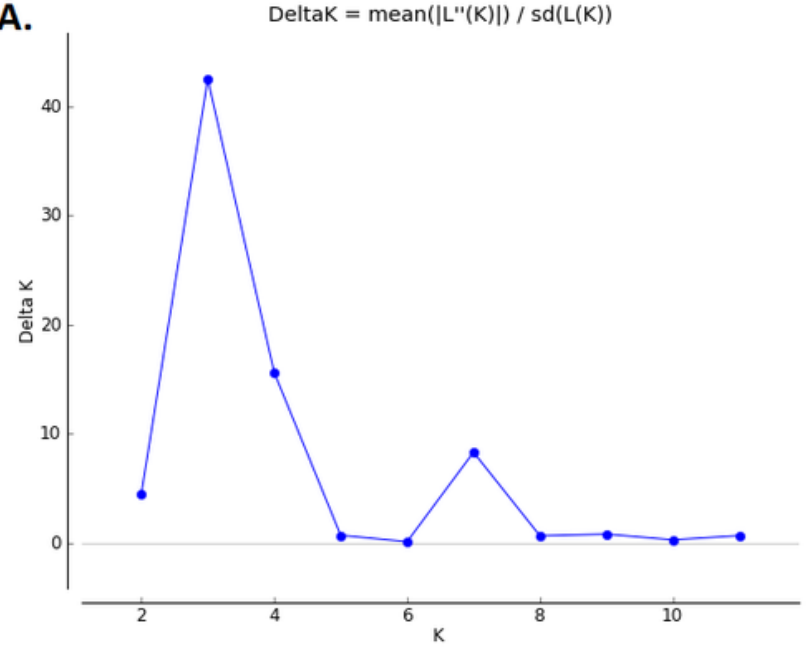

B.

$\mathrm{K}=2$

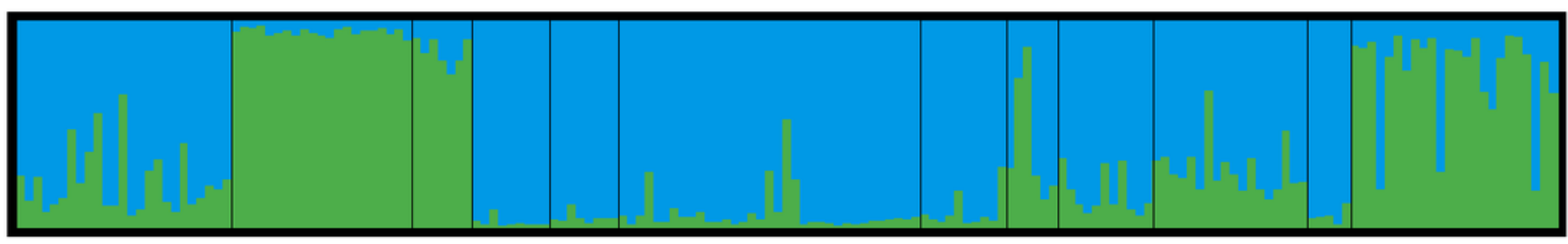

$K=3$

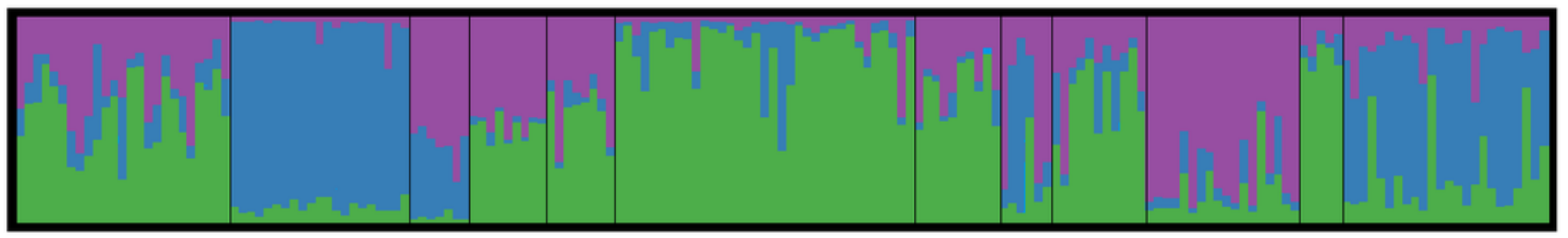

$K=4$

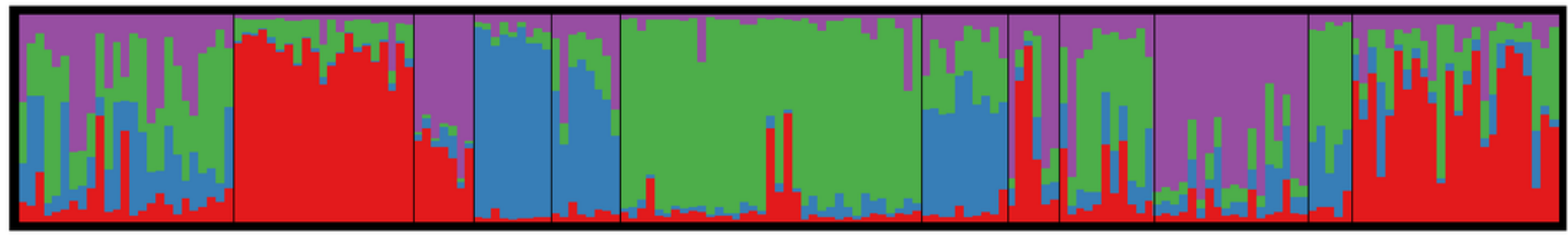

के

๙

iv

$0^{+} \omega^{\pi^{2}} 5^{5}$

$2 \pi e^{2}$ 